\title{
An Estimate of the Order of Magnitude of the Explosion During a Core Meltdown-Compaction Accident for Heavy Liquid Metal Fast Reactors: A disquieting result updating the Bethe-Tait model
}

\author{
Francisco J. Arias* and Geoffrey T. Parks* \\ Department of Engineering, University of Cambridge \\ Trumpington Street, Cambridge, CB2 1PZ, United Kingdom
}

(Dated: November 5, 2014)

\begin{abstract}
Criticality and recriticality considerations in heavy liquid metal fast reactors (HLMFRs) after a hypothetical core meltdown accident are discussed. Although many aspects of system behaviour in such scenarios can be deduced directly from the classical theory of sodium-cooled fast reactors (SFRs), certain ideas that have been accepted as true for SFRs cannot be extrapolated to HLMFRs without sufficiently careful thought. In this paper, we are concerned, as in SFRs, with fuel compaction, but with one important difference: there would be no boiling of the surrounding heavy liquid metal pool. Utilizing a Bethe-Tait model, it is shown that, due to the power flattening effect of the heavy liquid metal, explosive excursions at least an order of magnitude higher than for SFRs in similar situations are conceivable.
\end{abstract}

Keywords. hypothetical core disruptive accidents, heavy liquid metal reactor, recriticality. disassembly phase, Bethe-Tait model

\section{INTRODUCTION}

Although many aspects of the severe accident performance of heavy liquid metal fast reactors (HLMFRs) can be inferred from past work on the safety analysis of sodium-cooled fast reactors (SFRs), there are particular aspects of HLMFR behaviour that cannot be predicted or extrapolated based on SFR behaviour without sufficiently careful thought. This paper considers some particular situations during the so-called disassembly phase of core disruptive accidents, and specifically the compaction of fuel in the presence of the surrounding heavy liquid metal pool after a hypothetical core meltdown accident, but with one important difference in comparison with SFRs: there would be no boiling. The lack of boiling in this scenario has been claimed as a point in favour of HLM technology; however, as will be demonstrated, it results in disquieting and serious consequences during a core meltdown accident. This paper seeks to assess these consequences.

The present analysis should not be misunderstood as an attempt to provide a definitive analysis of the scenario under consideration, or some indication of the probability of such an accident, but seen as a theoretical examination of a conceivable event in an HLMFR. In the same way, the likelihood of a loss of flow or an overpower transient with a failure of the plant protection system to scram the reactor are the subject of other studies and lie outside the scope of the present paper.

*Corresponding author: Tel.: +32 143321 94; Electronic address: fja30@cam.ac.uk;-gtp10@cam.ac.uk

\section{A. A brief review of the theory of nuclear} explosions in SFRs

Early work in SFR safety analysis was motivated by and based on an assumed core compaction that yielded a high rate of reactivity gain and led to physical disassembly of the core due to the build-up of internal core pressures (Waltar and Reynolds, 1981). The calculation of energy generation during this phase was originally based on the classic Bethe-Tait analysis (Bethe and Tait, 1956), and this approach has long prevailed as the basic guide to containment design for fast breeder reactor (FBR) systems. Although more sophisticated models have been developed, the Bethe-Tait approach correctly predicts the dependence of the disassembly reactivity on the reactor parameters, and thus will be used in this paper to calculate the effect of the unboiled heavy liquid metal during the disassembly phase.

Let us consider a loss of flow transient. This design basis accident (DBA) assumes multiple failures whereby the reactor coolant recirculation pumps stop accidentally while the reactor is at full power, without scram, so that the fission chain reaction is not stopped. The reduced coolant flow causes the coolant in the core to heat up and boil out of the core, thereby allowing the fuel to heat up (see Fig. 1, steps 2 and 3). The expulsion of the coolant, or fuel compaction by melting, would then raise the reactivity and generate an explosive power excursion. The U.S. Nuclear Regulatory Commission (NRC) estimated the explosive yield resulting from this particular accident to be equivalent to that from $500 \mathrm{~kg}$ of TNT (Webb, 1976).

The explosion mechanism is a sodium coolant vapor explosion that is assumed to occur when the molten fuel, which is created by the first mild power excursion, eventually interacts with the (Webb, 1976), and would occur 
1

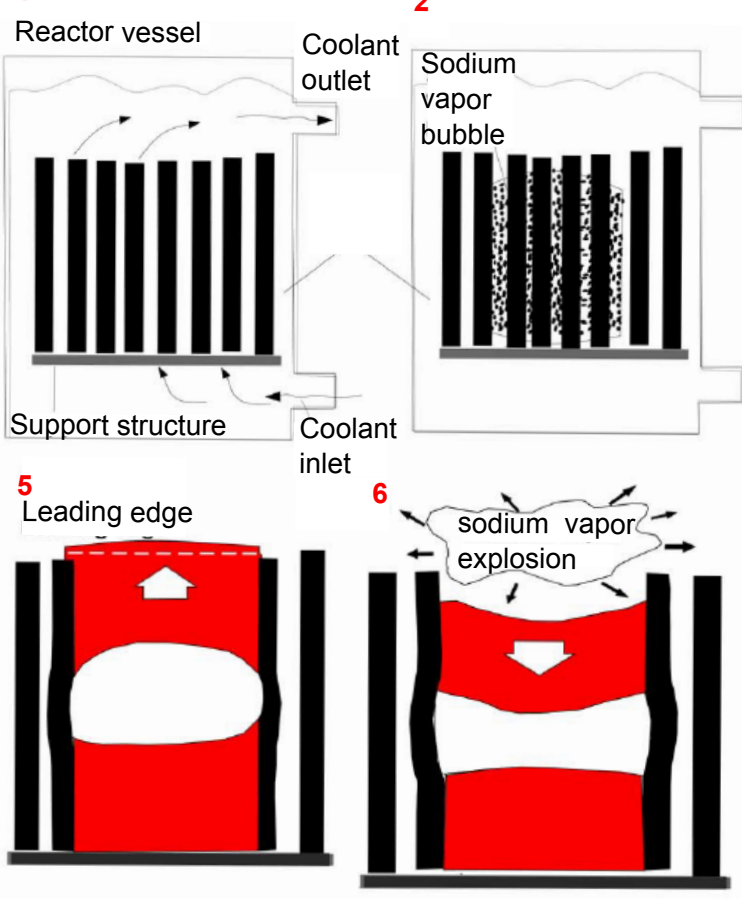

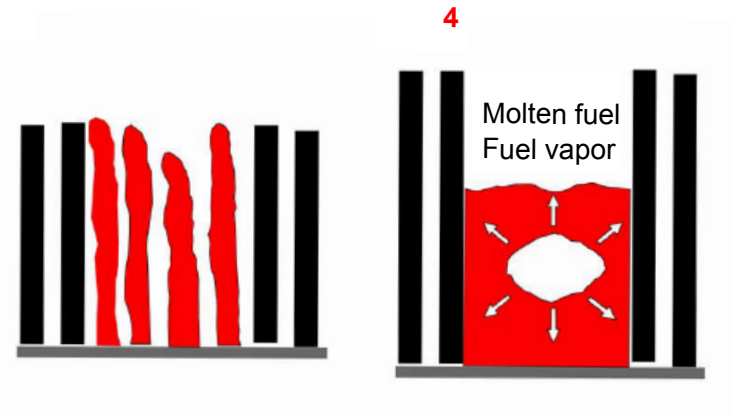

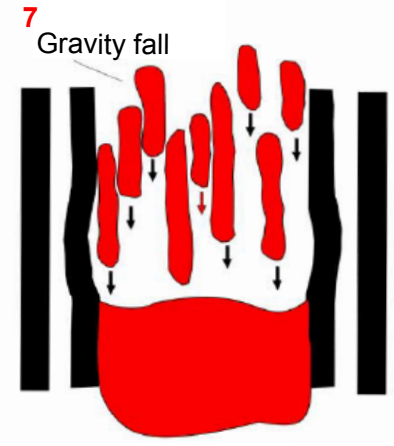

8

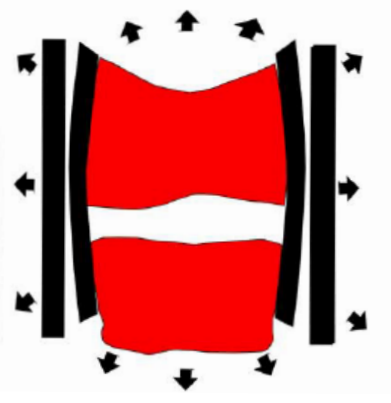

FIG. 1: A summary of the sequence of events in the disassembly phase for a SFR accident. Figure courtesy of Dr R.E. Webb (Webb, 1976). (1). Reactor at start of accident (coolant flow decreases);(2) core overheats; sodium coolant boils out of core; (3) core melts, slumps, compacts and rising reactivity; (4) mild power and fuel vapor explosion; (5) part of core thrown upward, leading edge interacts with sodium; (6) sodium vapor occurs, which propels part of the core downward to rejoin the lower part of the core, causing rapid fuel reassembling; (7) staggered (noncoherent model) fuel reentry, slow gravity fall, recriticality;(8) coherent mode secondary power excursion, about 5 tons TNT-equiv. nuclear explosion.

as follows: The power excursion of the DBA described above melts the core and produces a relatively mild fuel vapor explosion within the core; molten fuel from the core would then be blown (thrown up) into the coolant chamber above the core. The leading edge of the molten fuel entering the coolant chamber could, by mixing with coolant, generate a sodium vapor explosion. The force of the explosion (say, about $5 \mathrm{~kg}$ TNT-equivalent) could then drive a mass of fuel back down into the core, rapidly raising the reactivity by reassembling the fuel, to generate a disastrous secondary power excursion of about 5 tons TNT-equivalent. Such an explosion would vaporize the plutonium fuel and eject part of it into the atmosphere (see Fig. 1, steps 6-8).

\section{COMPACTION OF MOLTEN FUEL IN THE PRESENCE OF A HEAVY LIQUID METAL POOL WITH NO BOILING}

As liquid metals, liquid sodium $(\mathrm{Na})$, lead $(\mathrm{Pb})$ and lead-bismuth $(\mathrm{PbBi})$ are characterized by high thermal conductivities and low Prandtl numbers (Pr). Additionally, the high surface tension for $\mathrm{Pb}$ and $\mathrm{PbBi}$ leads to a situation in which most of the coolant in the core is super- heated before boiling is initiated. Hence, once a bubble does form, most of the superheated heavy liquid metal may be instantaneously transformed into vapor. In summary, we can say that it is highly unlikely that a sufficient level of superheating could be reached to initiate boiling with the immersion of molten fuel into a $\mathrm{Pb}$ or $\mathrm{PbBi}$ liquid pool while the bulk heavy liquid metal temperature is still substantially subcooled. The latent heat of evaporation for $\mathrm{Pb}$ and $\mathrm{PbBi}$ is large and the liquid would have to be superheated by $\gg 500^{\circ} \mathrm{C}$ before all the liquid could instantaneously vaporize. A substantial amount of energy is required to boil the heavy liquid metal in the pool (for a small-to-medium pool reactor this could be in the order of 1 GJ). So much energy would be not available in the early stages of the disassembly phase of an accident.

In this scenario, considering the sudden contact between the hot molten fuel and the cold heavy liquid metal coolant, the contact temperature $T_{i}$ will be established instantaneously according to

$$
\frac{T_{i}-T_{c}}{T_{h}-T_{i}}=\left(\frac{K_{h} m_{h} C_{h}}{K_{c} m_{c} C_{c}}\right)^{1 / 2}
$$

where $K$ is the thermal conductivity, $m$ the density, and $C$ the specific heat capacity, and subscripts $h$ and $c$ refer 


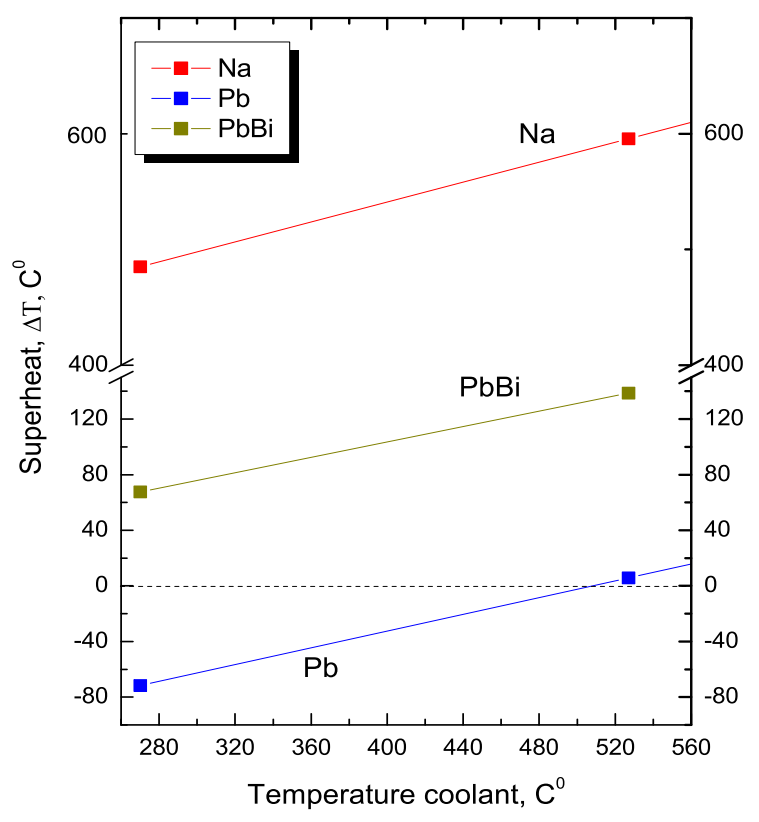

FIG. 2: Available superheat $\Delta T$ after sudden contact between molten fuel at a temperature $T_{h}=2950 \mathrm{~K}$ and liquid metal coolants, as a function of coolant temperature, for $\mathrm{Na}, \mathrm{Pb}$ and PbBi.

to the hot and cold liquids, respectively.

Fig. 2 shows the available superheat from instantaneous contact according to Eq. (1) for different surrounding liquids ( $\mathrm{Na}, \mathrm{Pb}$ and $\mathrm{PbBi}$ ), assuming: $T_{h}=2950 \mathrm{~K}$, the melting temperature of MOX $(0.7 \mathrm{U}-0.3 \mathrm{Pu}) \mathrm{O}_{2}$ fuel [1]; $m_{h} \approx 10000 \mathrm{kgm}^{-3} ; K_{h}=3 \mathrm{~W} / \mathrm{mK}: C_{h}=$ $450 \mathrm{~J} / \mathrm{kgK}$. It is clear that for the case of a heavy liquid metal $(\mathrm{Pb}$ or $\mathrm{PbBi})$, the fuel temperature would not be sufficiently beyond the saturation temperature $T_{\text {sat }}$ to initiate bubble nucleation for pool boiling. Finally, it should also be noted that radiative heat transfer could be strongly reduced in the case of $\mathrm{Pb}$ because of its low coefficient of emissivity $\varepsilon$ (see Appendix A).

\section{THE BETHE-TAIT MODEL}

The analytical method of calculating the energy release in a large nuclear burst in fast reactors given by Bethe-Tait (Bethe and Tait, 1956) assumes an exponential rise in the power. From this expression for the generated energy, an equation of state is used to calculate pressures, neglecting local expansion. Then, using the pressures obtained and neglecting propagation, displacements and reactivity reductions are calculated. The Bethe-Tait method, although based on some simplifying assumptions, correctly predicts the dependence of the disassembly reactivity on the other reactor parameters.

The following expression for the disassembly reactivity is deduced (Lewis 1977) within the framework of BetheTait model:

$$
\frac{d^{2} \rho_{d}}{d t^{2}}=-\frac{\bar{M}^{2}}{\bar{m} \bar{\kappa}_{\infty}\left\langle\psi_{0}^{2}\right\rangle}\left\langle p \nabla^{2}\left[\psi_{0} \nabla^{2} \psi_{0}-\left(\nabla \psi_{0}\right)^{2}\right]\right\rangle
$$

where $\rho_{d}$ is the disassembly reactivity, $\bar{M}$ is the average migration length, $\bar{m}$ the average core density, $\bar{\kappa}_{\infty}$ the infinite multiplication factor, $p$ the pressure, and $\psi_{0}$ is the spatial distribution of the power.

Assuming that the reactor is a bare, uniform sphere of radius $\bar{R}$. The spatial distribution of the power is then given by:

$$
\psi_{0}(r)=\frac{\bar{R}}{\pi r} \sin \left(\frac{\pi r}{\bar{R}}\right)
$$

where $r$ is the radial distance. For this flux distribution it may be shown that

$$
\nabla^{2}\left[\psi_{0} \nabla^{2} \psi_{0}-\left(\nabla \psi_{0}\right)^{2}\right]=\frac{4}{3} \frac{\pi^{4}}{\bar{R}^{4}}+O\left(r^{2}\right)
$$

Since it is expected that the pressure build-up will be concentrated near the center of the core, where $r$ is small, Eq. (3) can be approximated using a Taylor series:

$$
\psi_{0} \approx 1-\frac{\pi^{2} r^{2}}{6 \bar{R}^{2}}+\ldots
$$

whence Eq. (2) becomes:

$$
\frac{d^{2} \rho_{d}}{d t^{2}}=-\frac{8}{9} \frac{\pi^{6} \bar{M}^{2}}{\bar{m} \bar{\kappa}_{\infty} \bar{R}^{4}} \frac{1}{V}\langle p\rangle
$$

where $V$ is the volume of reactor, which for this model is equal to $\frac{4}{3} \pi \bar{R}^{3}$.

In Eq. (6), we now need an equation of state that allows the pressure to be connected with the energy density. Assuming a classical approach in which the pressure increases linearly with energy density above some threshold in energy density $e_{*}$, we have:

$$
\begin{array}{ll}
e<e_{*}: & p=0 \\
e \geq e_{*}: & p=(\gamma-1) \bar{m}\left(e-e_{*}\right),
\end{array}
$$

where $\gamma$ is the ratio of specific heats, and $e$ is the energy generated per unit mass of the core.

Assume that $e(r, t)$ is separable in space and time:

$$
e(r, t)=T(t) N(r)
$$

with the normalization condition:

$$
\langle N(r) m\rangle=1
$$

Combining Eqs. (7), (8) and (9), and inserting into Eq. (6), while also using both the relation between power $P$ and reactivity $\rho$ :

$$
\frac{d P(t)}{d t}=\frac{\dot{\rho} t}{\Lambda} P(t)
$$


where $\Lambda$ is the prompt neutron generation time, and the constant density approximation to the momentum equation:

$$
\frac{\partial \mathbf{v}}{\partial t}=-\frac{1}{\bar{m}} \nabla p
$$

where $\mathbf{v}$ is the velocity, we obtain, after a series of calculations, the total energy release $E$ into the Bethe-Tait model, as (Lewis 1977):

$$
E \approx \frac{9}{8} \frac{\bar{\kappa}_{\infty} \bar{m}}{\pi^{6} \bar{M}^{2}(\gamma-1)}\left(\ln \left\{\frac{\dot{\rho}}{\Lambda}\left[\frac{E_{*}}{P(0)}\right]^{2}\right\}\right)^{3 / 2} \bar{R}^{4} V \frac{\dot{\rho}^{3 / 2}}{\Lambda^{1 / 2}}
$$

where $E_{*}$ is the energy at the initiation of disassembly, and $P_{0}$ is the power level at the moment the reactor is brought prompt critical (at $t=0$ ).

Now, in the particular application under consideration, the heavy liquid metal surrounding the core acts as a reflector. Thus, using the subscripts $c$ and $r$ to denote core and reflector respectively, the following differential equations governing the neutron flux distribution can be defined:

$$
\begin{gathered}
D_{c} \nabla^{2} \psi_{c}-\Sigma_{c} \psi_{c}+\kappa \Sigma_{c} \psi_{c}=0 \\
D_{r} \nabla^{2} \psi_{r}-\Sigma_{r} \psi_{r}=0
\end{gathered}
$$

where $D$ is the diffusion coefficient, $\Sigma$ is the macroscopic absorption cross-section, and no source term appears in Eq. (14) (the reflector equation) since the liquid metal coolant is presumed not to be fissionable.

The solution of Eq. (13) (the equation for the core) is:

$$
\psi_{c}(r)=\frac{A \sin B_{c} r}{r}
$$

and the solution of Eq. (14) with the assumption that $R_{c} \rightarrow \infty$ :

$$
\psi_{r}(r)=\frac{A^{\prime} e^{-r / L_{r}}}{r}
$$

where

$$
L_{r}^{2}=\frac{D_{r}}{\Sigma_{r}}
$$

Recognising that the neutron flux $\psi$ and current $J$ must be continuous across the interface between core and reflector and therefore:

$$
\begin{aligned}
& \psi_{c}(\bar{R})=\psi_{r}(\bar{R}) \\
& J_{c}(\bar{R})=J_{r}(\bar{R})
\end{aligned}
$$

then establishes the following system of equations:

$$
\frac{A \sin B_{c} \bar{R}}{\bar{R}}=\frac{A^{\prime} e^{-\bar{R} / L_{r}}}{\bar{R}}
$$

$$
\begin{aligned}
-D_{c} A\left(\frac{B_{c} \cos B_{c} \bar{R}}{\bar{R}}\right. & \left.-\frac{\sin B_{c} \bar{R}}{\bar{R}^{2}}\right) \\
& =D_{r} A^{\prime} e^{-\bar{R} / L_{r}}\left(\frac{1}{L_{r} \bar{R}}+\frac{1}{\bar{R}^{2}}\right)
\end{aligned}
$$

Combining these equations and simplifying:

$$
B_{c} \bar{R} \cot B_{c} \bar{R}=1-\frac{D_{r}}{D_{c}}\left(1+\frac{\bar{R}}{L_{r}}\right)
$$

assuming $D_{r} \approx D_{c}$, which is not a strong approximation for $\mathrm{Pb}$ and $\mathrm{PbBi}$ :

$$
\cot B_{c} \bar{R}=-\frac{1}{B_{c} L_{r}}
$$

resulting in two special cases, namely:

- assuming high absorption in the reflector, and

- assuming negligible absorption in the reflector.

For the first case, the core buckling is then

$$
B_{c}=\frac{\pi}{\bar{R}}
$$

which is, of course, the same expression as for a voided core. For the case of negligible absorption in the reflector, we have:

$$
B_{c}=\frac{\pi}{2 \bar{R}}
$$

For the sake of simplicity, a generalized expression may be used to take into account the effect of the reflector on the core buckling:

$$
B_{c}=\frac{\pi}{f \bar{R}}
$$

where $\boldsymbol{f}$ is the power flattening factor, the value of which lies between $f=1$ for a reflector with high absorption or a voided core, and $\boldsymbol{f}=2$ for a reflector with low neutronic absorption.

Thus, proceeding in the same way in calculating the yield of available energy but considering, instead of Eq. (3) for the spatial distribution of the power, the calculated effect of the power flattening of reflector:

$$
\psi_{0}=\frac{f \bar{R}}{\pi r} \sin \left(\frac{\pi r}{f \bar{R}}\right)
$$

the final available energy $E$ can be expressed as:

$$
\frac{E}{E_{0}}=\left[f^{6}-\frac{f^{7}}{2 \pi} \sin \frac{2 \pi}{f}\right]
$$

where $E_{0}$ is the available energy in the absence of a reflector, which is given by Eq. (12).

In the limit as $\boldsymbol{f} \rightarrow 2$, Eq. (28) gives the upper bound on $E$ as:

$$
\frac{E}{E_{0}} \approx f^{6}
$$


To obtain an estimate of the parameter $\boldsymbol{f}$ for a leadcooled reactor, we can consider the core buckling

$$
B_{c}^{2}=\frac{\kappa_{\infty}-1}{L_{c}^{2}}
$$

where $\kappa_{\infty}$ is the infinite multiplication factor, and

$$
L_{c}^{2}=\frac{D_{c}}{\Sigma_{c}}
$$

Then, inserting Eqs. (31), (30) and (17) into Eq. (23) yields:

$$
\cot B_{c} \bar{R}=-\frac{1}{\sqrt{\kappa_{\infty}-1}} \frac{\Sigma_{r}}{\Sigma_{c}}
$$

Assuming $\kappa_{\infty} \approx 1.8$, and $\Sigma_{r} / \Sigma_{c} \approx 0.01$, we obtain that $f \approx 1.98$.

If it is acceptable to ignore the mild effects of the logarithmic term in Eq. (12), an "effective" rate of reactivity insertion may be defined as

$$
\dot{\rho}_{\mathrm{eff}}=\dot{\rho} \cdot f^{4}
$$

Although Eq. (29) suggests the reflector has a strong effect on the energy release, a weaker dependence could be anticipated considering the approximations used. For example, in Eq. (5) using a Taylor series expansion, we are neglecting the dependence of $\rho_{d}$ on the power distribution in the core. According to Jankus (Jankus. 1962) a parabolic distribution results in a weaker dependence on the power flattening factor for large excursions of the form:

$$
\frac{E-E_{*}}{E_{*}} \longrightarrow \frac{q^{-3 / 2}}{1-0.6 q} \cdot \frac{1-\frac{6 q}{5}+\frac{3 q^{2}}{7}}{q^{1 / 2}}
$$

where $q=\frac{\pi^{2}}{6 f^{2}}$. Fig. 3 shows the ratio of excess energy to threshold energy, $\frac{E-E_{*}}{E_{*}}$, as a function of the power flattening factor $\boldsymbol{f}$ according to Eq. (34). This shows that the presence of a reflector could enhance the explosion yield by up to an order of magnitude.

\section{DOPPLER EFFECT}

Thus far, we have deduced the strong effect of the presence of the surrounding unboiled heavy liquid metal on the magnitude of disassembly reactivity, and then on the increase in the explosive yield of a power excursion. These calculations show that an increase in explosive yield of up to an order of magnitude is theoretically conceivable. However, no Doppler feedback was considered in these calculations. Doppler feedback could be, as is well known, an important mitigating mechanism.

For scoping calculations of this sort, it would be useful to be able to deduce the value of Doppler coefficient that will cause the explosive release to become, say, an

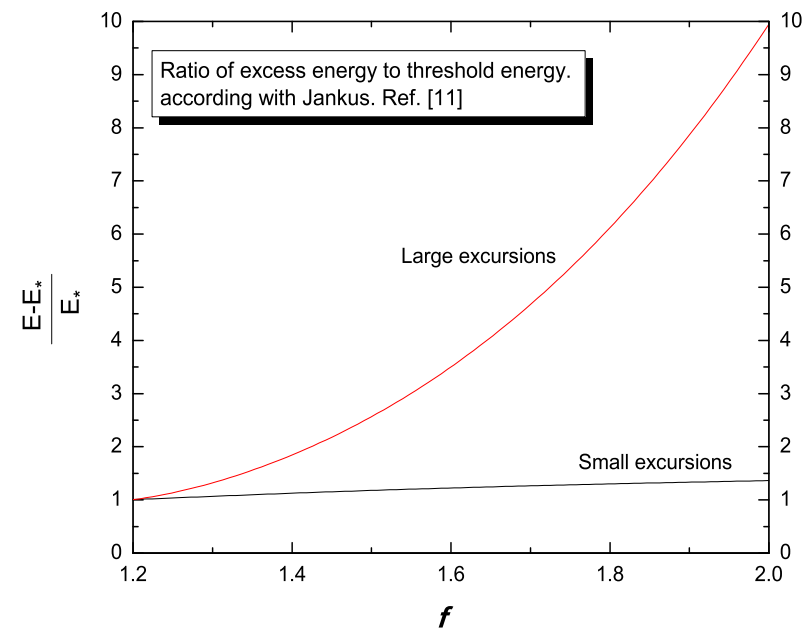

FIG. 3: The ratio of excess energy to threshold energy, $\frac{E-E_{*}}{E_{*}}$, as a function of the power flattening factor $f$ in the simple Bethe-Tait calculation according to Jankus (Jankus. 1962).

order of magnitude smaller, mitigating the effect of the surrounding heavy liquid metal. Smith et al. (Smith, et al. 1965) estimated the critical value of the Doppler coefficient that will cause the explosive release to become one order of magnitude less than that which would occur in the absence of prompt reactivity feedback. An estimate of the Doppler coefficient needed to meet this criterion is (Smith, et al. 1965):

$$
K_{D}=-\frac{\left[2 \dot{\rho} \Lambda \ln \left(e_{*} \dot{\rho} \sqrt{\left(2 / \pi \beta P_{0}\right)}\right)\right]^{1 / 2}}{\ln \left[1+0.73\left(e_{*} / c_{v} T_{0}\right)\right)}
$$

where $P_{0}$ is the reactor power at delayed critical, $T_{0}$ is the initial average fuel temperature, $\beta$ is the delayed neutron fraction, and $c_{v}$ is the specific heat capacity at constant volume.

Thus, for a small (say $100 \mathrm{MWth}$ ) fast reactor with the following typical parameter values: $\gamma=2 ; e_{*}=10^{6} \mathrm{~J} / \mathrm{kg}$; $T_{0}=1800 \mathrm{~K} ; c_{v}=300 \mathrm{~J} / \mathrm{kgK} ; \Lambda=5 \times 10^{-7} \mathrm{~s} ; \beta=$ $0.3 \% ; P_{0}=2.5 \times 10^{-2} \mathrm{MW} / \mathrm{kg}$; we obtain the relationship shown in Fig. 4. For an initial rate of change of reactivity of $\dot{\rho}=100 \$ / \mathrm{s}$, a Doppler coefficient of $-370 \mathrm{pcm}$ will reduce the explosive yield by one order of magnitude.

In applying the above results, ANL calculated a nuclear explosion of about $655 \mathrm{~kg}$ TNT-equivalent for the accident in the Fast Flux Test Facility (FFTF) reactor with a reactivity insertion of $100 \$ / \mathrm{s}$ [24]. For the same reactivity insertion of $100 \$ / \mathrm{s}$ applied to a small (100 MWth) fast reactor the $655 \mathrm{~kg}$ TNT-equivalent figure would scale down to a $\sim 60 \mathrm{~kg}$ TNT-equivalent explosion considering the $\sim 70$ fuel assemblies and neglecting the effect of the surrounding heavy liquid metal. Accounting for the presence of the unboiled heavy liquid metal pool according to Eq. (29) and taking $\boldsymbol{f} \approx 2$ predicts a 


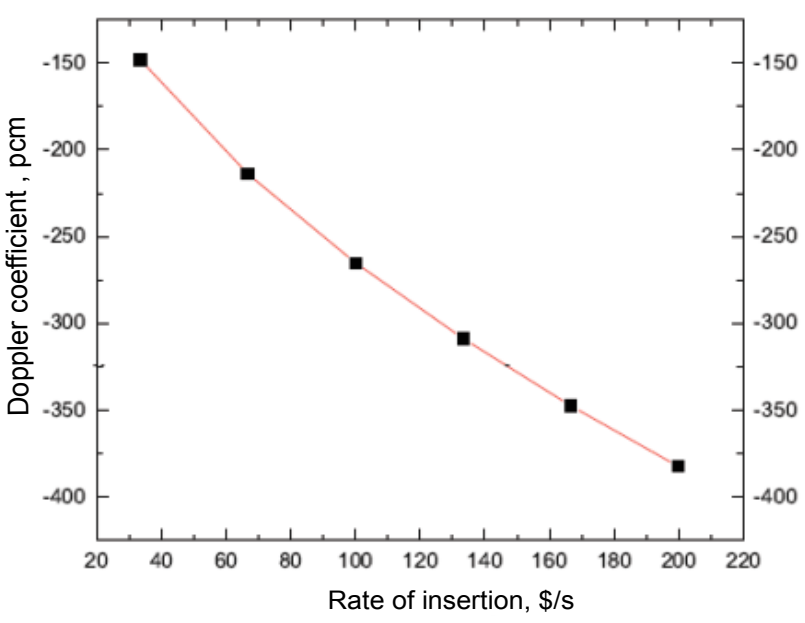

FIG. 4: Doppler coefficient needed to reduce the energy release by one order of magnitude as a function of initial reactivity insertion rate.

3.8 ton TNT-equivalent explosion, or something like 6.4 World War II 'blockbuster' bombs, exceeding the maximum economically viable explosion containment capability.

\section{THE PERFORMANCE OF THE VESSEL}

A TNT explosion is a detonation lasting only microseconds, creating local pressures of the order of 50,000 atmospheres. However, the hypothesized energy release from a nuclear excursion resembles that from propellant-type conflagrations, creating pressures of only a few hundred atmospheres but lasting over a timescale of milliseconds. The forms of the energy release and of the resulting structural damage differ significantly between a high explosive detonation and a propellant conflagration.

Considering the yield of TNT Containment Law for ideal vessels (nozzles, weldments, or rigid end constraints) given by (Wise and Proctor 1965):

$$
W=\left[\frac{1.407 \sigma_{t} \epsilon\left(3.41+0.117 R_{i} / h_{0}\right)\left(R_{e}^{2}-R_{i}^{2}\right)^{1.85}}{10^{5} m^{-0.85}\left(1.47+0.0373 R_{1} / h_{0}\right)^{0.15} R_{i}^{0.15}}\right]^{0.811}
$$

where $W$ is the charge weight (of TNT or pentolite) in lb, $m$ is the density of the vessel material in $\mathrm{lb} / \mathrm{ft}^{3}, R_{i}$ is the initial internal radius of the vessel in $\mathrm{ft}, R_{e}$ is the initial external radius of the vessel in $\mathrm{ft}, h_{0}$ is the initial wall thickness of the vessel in $\mathrm{ft}, \epsilon$ is the maximum permissible strain of the vessel material, and $\sigma_{t}$ is the true stress in psi, given by:

$$
\sigma_{t}=\sigma_{y}+\left[\frac{\sigma_{u}\left(1+\epsilon_{u}\right)-\sigma_{y}}{\epsilon_{u}}\right] \epsilon
$$

where $\sigma_{y}$ is the yield stress of the vessel material in psi, $\sigma_{u}$ is the ultimate stress of the vessel material in psi, and $\epsilon_{u}$ is the ultimate strain of the vessel material.

For vessels fitted with nozzles, weldments, and rigid end closures, it was postulated that real vessels can be expected to withstand safely strains as large as $\frac{1}{3} \epsilon_{u}$. Imposing this restriction on Eq. (36) yields:

$$
W=\left[\frac{0.1563 m^{0.85} \sigma_{u} \epsilon_{u}\left(3.41+0.117 R_{i} / h_{0}\right)\left(R_{e}^{2}-R_{i}^{2}\right)^{1.85}}{10^{5}\left(2 \sigma_{y}+\sigma_{u}+\sigma_{u} \epsilon_{u}\right)^{-1}\left(1.47+0.0373 R_{i} / h_{0}\right)^{0.15} R_{i}^{0.15}}\right]^{0.811}
$$

Applying this equation, with the following parameters for the vessel of a typical small (100 MWth) reactor: $m=$ $8000 \mathrm{~kg} / \mathrm{m}^{3}, R_{i}=4 \mathrm{~m}, R_{e}=4.08 \mathrm{~m}, h_{0}=8 \mathrm{~cm}, \epsilon_{u}=$ $0.5, \sigma_{t}=472.33 \mathrm{MPa}, \sigma_{y}=290 \mathrm{MPa}, \sigma_{u}=588 \mathrm{MPa}$, we obtain an upper bound for the maximum containment capability of about $422 \mathrm{~kg}$ TNT-equivalent.

Taking into account our previous calculations, for a full gravitational compaction of the 100 MWth core, the vessel could withstand the $60 \mathrm{~kg}$ TNT-equivalent explosion from a $100 \$ / \mathrm{s}$ reactivity insertion if a bare core is assumed; however, allowing for the presence of the unboiled heavy liquid metal coolant, the explosive yield would be on the order of 3.8 ton TNT-equivalent according to Eq. (29) with $\boldsymbol{f}=2$ or about $600 \mathrm{~kg}$ TNT-equivalent with the weaker dependence from Jankus (Jankus. 1962), see Eq. (34).

\section{SUMMARY OF RESULTS AND CONCLUSIONS}

A methodology for providing an estimate of the order of magnitude of the explosion during a core meltdowncompaction accident for heavy liquid metal fast reactors has been presented. Utilizing the Bethe-Tait model, it is shown that a much more powerful explosion is conceivable owing to the possibility of the presence of unboiled heavy liquid metal during the compaction. Taking into account the reduction due to the Doppler effect, the explosive yield could be at least one order of magnitude higher for lead or lead-bismuth cooled fast reactors than for sodium fast reactors. In light of this disquieting result, additional research and development is required to investigate further this accident scenario. 


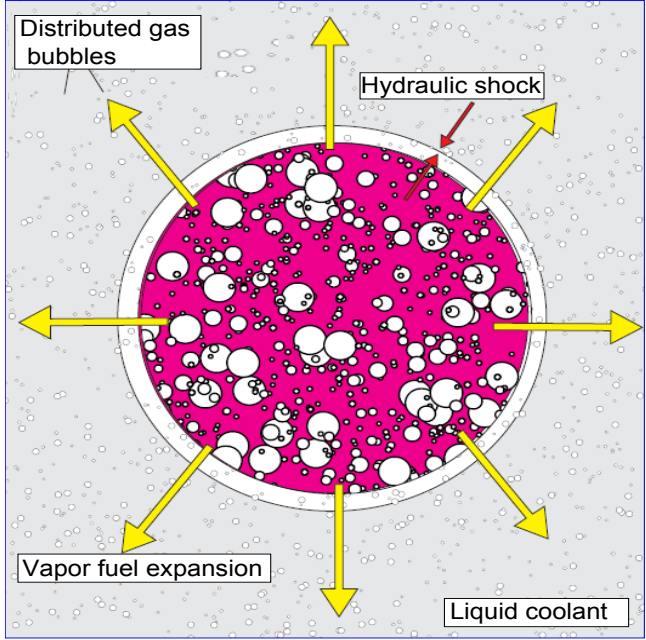

FIG. 5: Core disassembly model for a hard system

\section{APPENDIX A: EMISSIVITY IN A LEAD-SHELL BUBBLE}

Let us assume that the spherical volume of mixed gases (lead and fuel) is surrounding by liquid lead media, representing two parallel layers whose facing surfaces have emissivities $\varepsilon_{f}$ and $\varepsilon_{P b}$.

A certain fraction of the radiation of a specific wavelength leaves the first internal layer (with emissivity $\varepsilon_{f}$ ) and enters the second external layer (with emissivity $\left.\varepsilon_{P b}\right)$. A fraction of this incident radiation does not pass through the surface to the other side and is reflected.

The fraction of the radiation that is reflected back to the first layer will be $\varepsilon_{f}\left(1-\varepsilon_{P b}\right)$. Then, a fraction equal to $\varepsilon_{f}\left(1-\varepsilon_{P b}\right)\left(1-\varepsilon_{f}\right)$ will be reflected back to the second layer again. Due to this process of back and forth reflection, a steady state results giving the total proportion of radiation entering the second layer (from the first layer) to be:

$$
\varepsilon_{f, P b}=\frac{\varepsilon_{f} \varepsilon_{P b}}{\varepsilon_{f}+\varepsilon_{P b}\left(1-\varepsilon_{f}\right)}
$$

If the first layer has an ideal emissivity, i.e. $\varepsilon_{f}=1$, and the second layer has an emissivity $\varepsilon_{P b} \ll \varepsilon_{f}$ then $\varepsilon_{f, P b} \approx \varepsilon_{P b}$.

Wien's Law for a gas at a temperature $T$ of $3500 \mathrm{~K}$ gives a maximum radiation wavelength on the order of $\mu \mathrm{m}$ at which the emissivity of lead is 0.05 .

\section{APPENDIX B: EQUATION OF STATE}

In order to contemplate the possibility of a single-phase or "hard-system", we can deduce a rudimentary expression to predict the suitability of this pressure.

Let us consider that after a certain expansion, the vapor fuel hit the surrounding liquid lead and stop instantaneously in a very short time which is compared with the propagation time for a pressure wave to travel the length of the core. (See Fig. 9)

The pressure pulse produced by the instantaneously desacceleration of the expanded vapor fuel can be calculated from the Joukowsky equation,

$$
\frac{\delta p}{\delta t}=\bar{m} c \frac{\delta v}{\delta t}
$$

where, here, $\bar{m}$ is the average fuel density, $c$ is the speed of sound in the fluid, and $v$ is the velocity of expanded fuel, and $t$ the time interval. Considering the assumption of suddenly des-acceleration the above expression may be rewritten as

$$
p=\bar{m} c v
$$

However, the speed of sound, $c$, is dependent upon the physical properties of the liquid, and the presence of bubbles markedly decreases the effective speed of sound in the liquid. Although the rigorous treatment of the acoustic characteristics of bubbles requires a great deal of rather advanced mathematics, (Epstein 1941), (Foldy. 1944). However, a simple approximation can be made when the radius $R$ of the bubbles is very small compared with the wavelength of sound in the liquid, or (Research Analysis Group, 1969.)

$$
\eta=\frac{2 \pi R}{\lambda} \ll 1
$$

where $\eta$ is the ratio of the bubble circumference to the wavelength . This lead to the equation

$$
\frac{c_{0}^{2}}{c^{2}}=1+\frac{3 \phi}{\eta_{r}^{2}}
$$

where $c_{0}$ is the speed of sound when no bubbles are present, and $\eta_{r}$ is is the ratio of the bubble circumference $2 \pi R$ to the wavelength $\lambda$ at resonance, defined as

$$
\eta_{r}=\frac{1}{c} \sqrt{\frac{3 \gamma p_{0}}{\bar{m}}}
$$

being $p_{0}$ the pressure surrounding the bubbles. and $\phi$ is the total volume of of gas present as bubbles in $1 \mathrm{~cm}^{3}$ of the liquid-bubble mixture. Thus $\phi$ is defined by the equation

$$
\phi=\int \frac{4 \pi}{3} R^{3} n(R) d R
$$

where $n(R)$ s the bubble distribution. Assuming a homogeneous mixture of bubbles, then, $\phi$ may be written as function of distribute void fraction (percent) as

$$
\phi=\frac{\alpha}{100}
$$


The speed of sound in a liquid is given by

$$
c_{0}=\sqrt{\frac{\gamma(K+b \cdot p)}{\bar{m}}}
$$

where $K$ is the bulk modulus of the fluid, and $b$ is a constant that takes account the pressure-dependence with a value between $2-5$.

Taking into account that the velocity of expanded gas can be put as function of kinetic energy per unit of mass according with

$$
v=\sqrt{2 e}
$$

where $e$ is the energy per unit of mass. Upon substituting equations (B9),(B8) and (B4) into Eq. (B2), one obtain for the equation of state.

\begin{tabular}{|c|c|c|c|}
\hline & $\begin{array}{c}\text { Total } \\
\text { energy } \\
\text { Voids }\end{array}$ & $\begin{array}{c}\text { Final } \\
\text { Pressure } \\
\text { at Core } \\
\text { Center } \\
\text { (atm) }\end{array}$ & $\begin{array}{c}\text { Final } \\
\text { Temperature } \\
\text { at } \\
\text { Core Center } \\
\left({ }^{\circ} K\right)\end{array}$ \\
\hline $\begin{array}{c}\text { Distributed } \\
\text { Voids }\end{array}$ & & & \\
$(\%)$ & & & \\
0 & 563 & 3.8 & 4104 \\
0.2 & 704 & 6.4 & 4255 \\
0.5 & 905 & 12.3 & 4471 \\
1.0 & 1220 & 29.9 & 4810 \\
2.4 & 1810 & 111 & 5437 \\
5.0 & 2560 & 373 & 6233 \\
40.0 & 2620 & 399 & 6287 \\
Only central & 3160 & 739 & 6834 \\
zone voided & & & \\
Central zone & & & \\
voided and 1\% & 1860 & 123 & 5495 \\
void in blanket & & & \\
\hline
\end{tabular}

TABLE I: Dependence upon initial voids with insertion Rate of $100 \$ /$ sec, [13].

$$
p=2 \bar{m} \gamma\left(e-e_{*}\right)\left[\frac{K}{p}+b\right] \frac{1}{1+\frac{3 \alpha}{100 \eta_{r}^{2}}}
$$

Although with some excessive simplification, suffice it to point out the dramatic dependence with the distributed void because the strongly effect on the speed of sound. The above result seems in agreement with the calculations from (Nicholson and Jackson) in their computer calculations using a two-dimensional neutronichydrodynamics disassembly program (see Table I).

In Fig.5, to obtain some idea of the pressure predicted by Eq. (B10), we assume some typical values of the parameters for sodium: $b=3, \gamma=1.98$, sound speed of

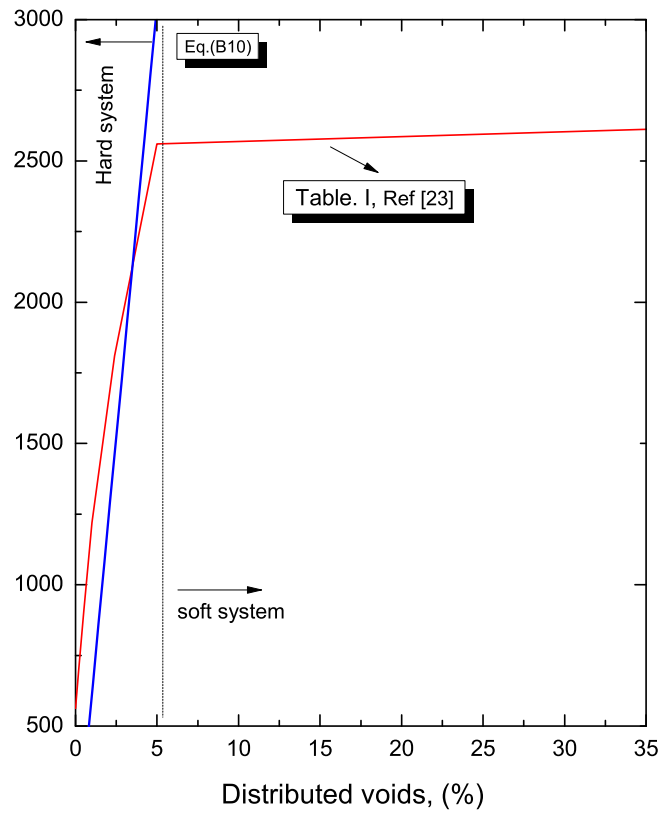

FIG. 6: Dependence total energy upon initial voids with insertion Rate of $100 \$ /$ sec for FFTF according with (Nicholson and Jackson)and Eq. (B10)

$1800 \mathrm{~m} / \mathrm{s}, p=1$ atm, $\eta_{r}=4 \cdot 10^{-2}$ in comparison with the calculations from (Nicholson and Jackson) and Eq. B10, where seems that from a threshold of bubble concentration the system behaves as a two-phase or "soft system"

\section{NOMENCLATURE}

$A=$ constant of integration

$b=$ constant

$B_{c}=$ core buckling

$D=$ diffusion coefficient

$c=$ speed of sound

$e=$ energy per unit mass

$\boldsymbol{f}=$ power flattening factor, Eq. (26)

$g=$ acceleration due to gravity

$J=$ neutron current

$K=$ bulk modulus of the fluid

$k=$ thermal conductivity

$t=$ time

$h_{0}=$ initial wall thickness of vessel

$L_{c}=$ cover gas length

$L=$ neutron path length

$M=$ migration length

$M_{f}=$ mass of fuel

$M_{P b B i}=$ Mass of fuel $\mathrm{PbBi}$ column above diaphragms of MYRRHA

$n=$ bubble distribution 
$N=$ time dependence of power flux

$m=$ density

$p=$ pressure

$P=$ power

$r=$ radial distance

$R_{i}=$ initial internal radius of vessel

$R_{e}=$ initial external radius of vessel

$R_{g}=$ ideal gas constant

$\bar{R}=$ radius of equivalent core

$v=$ velocity

$t=$ time

$W=$ charge weight

$z=$ depth penetration of the molten fuel into the heavy

liquid metal pool

\section{Greek symbols}

$\alpha=$ void fraction (\%)

$\rho=$ reactivity

$\kappa=$ multiplication factor

$\epsilon=$ maximum permissible strain of vessel material

$\epsilon_{u}=$ ultimate strain of vessel material

$\varepsilon=$ emissivity

$\psi=$ spatial distribution of the power

$\Lambda=$ prompt neutron generation time

$\gamma=$ ratio specific heats

$\Sigma=$ macroscopic cross-section

$\sigma_{t}=$ true stress of vessel material

$\sigma_{y}=$ yield stress of vessel material

$\sigma_{u}=$ ultimate stress of vessel material $\eta=$ ratio of the bubble circumference to the wavelength $\phi=$ total volume of gas present as bubbles in $1 \mathrm{~cm}^{3}$ of the liquid-bubble mixture

\section{Subscripts}

$\infty=$ infinite multiplication factor

$c=$ core

$d=$ disassembly

$r=$ reflector

$h=$ hot

$c=$ cold

$f=$ fuel

$l=$ liquid

$\mathrm{Pb}=$ lead

$\mathrm{PbBi}=$ lead-bismuth eutectic

\section{ACKNOWLEDGEMENTS}

The first author is indebted to engineer M. Scott for many helpful, encouraging, and stimulating discussions which led to greater understanding of the problem considered here.

REFERENCES
[1] Adamson, M.G., Aitken, E.A., Caputi, R.W., 1985. Experimental and Thermodynamic Evaluation of the Melting Behavior of Irradiated Oxide Fuels. J. Nuclear Mater. 130, 349-365.

[2] Bethe, H.A., Tait, J.H., 1956 An Estimate of the Order of Magnitude of the Explosion When the Core of a Fast Reactor Collapses. UKAEA-RHM.

[3] Belle, J., 1961. Uranium Dioxide: Properties and Nuclear Applications, U.S. Government Printing Office.

[4] Boudreau, J.E., 1974. Recriticality Considerations in LMFBR Accidents. Argonne National Laboratory, ANLLA-UR-74-520.

[5] Choe, H., Epstein, M., Fauske, H.K., 1974. Work Potential Resulting from a Voided Core Disassembly. Trans Am. Nucl. Soc. 18, 220.

[6] Epstein, S.P., 1941. On the Absorption of Sound Waves in Suspensions and Emulsions. Theodor von K'arman Anniversary Volume, CIT, pp. 162-168.

[7] Faupel, J.H., 1964. Engineering Design: Synthesis of Stress Analysis and Materials Engineering. John Wiley and Sons, Inc. New York.

[8] Foldy, L., 1944. Propagation of Sound Through a Liquid Containing Bubbles: Part I. General Theory. OSRD 3601, NDRC 6.1-sr1130-1378, Project NS-141, USRL. Div. 6-540.22-M2.

[9] Hicks, E.P., Menzies, D.C., 1965. Theoretical Studies on the Fast Reactor Maximum Accident. Proc. Conf. on Safety, Fuels and Core Design in Large Fast Power Reac- tors, Argonne National Laboratory Report, ANL-7120.

[10] Jankus, V.Z., 1962. A Theoretical Study of Destructive Nuclear Bursts in Fast Reactors. Argonne National Laboratory, ANL-6512.

[11] Lewis, E.E., 1977. Nuclear Power Reactor Safety. John Wiley and Sons, New York. Chapter 5.

[12] Meyer, R.A., Wolf, B., Friedman, N.F., Seifert, R., 1967. Fast Reactor Meltdown Accidents Using Bethe-Tait Analysis. General Electric Report. GEAP-4809.

[13] Nicholson, R.B., Jackson, J.F., 1971. Partial Voiding and Fission Product Gas in LMFBR Disassembly Calculations. Trans. Am. Nucl. Soc. 14(2), 737.

[14] Ostensen, R.W., Jackson, J.F., 1973. Extended Fuel Motion Study. In: Reactor Development Programme Progress Report. Argonne National Laboratory, ANLRDP-18.

[15] Proctor, J.F., 1967. Adequacy of Explosion-Response Data in Estimating Reactor-Vessel Damage. Nucl. Safety 8, 565-572.

[16] Rauh, E.G., Thorn, R.J., 1954. The Vapor Pressure of Uranium. J. Chem. Phys. 22, 1414.

[17] Research Analysis Group, 1969. Physics of Sound in the Sea, Chapter 28. Department of the Navy. Headquarters Naval Material Command, Washington, D.C.

[18] SL-1 Accident: Report, 1961. U.S. Atomic Energy Commission. SL-1 Accident Investigation Board. United States. Congress. Joint Committee on Atomic Energy.

[19] Smith, R.D., Rowlands, J.L., Baker, A.R., Smith, D.C., 
Hicks, E.P., Mann, J.E., Weale, J., 1965. Fast Reactor Physics, Including Results from UK Zero Power Reactors. Proc. Third United Nations Conference on Peaceful Uses of Atomic Energy. Vol. 6. United Nations, Geneva, 1964, New York, 1965.

[20] Suk, S-D., Hahn, D., 2002. Analysis of Core Disruptive Accident Energetics for Liquid Metal Reactor. J. Korean Nucl. Soc. 34, 117-131.

[21] Thompson, T.J, Beckerley, J.G., 1964. The Technology of Nuclear Reactor Safety, Vol. 1. MIT Press, Cambridge MA.
[22] Waltar, A.E., Reynolds, A.B., 1981. Fast Breeder Reactors. Pergamon Press, Elmsford, New York.

[23] Wirtz, K., 1973. Lectures on Fast Reactors. Kernforschungszentrum, Karlsruhe.

[24] Webb, R.E., 1976. The Accident Hazards of Nuclear Power. University of Massachusetts Press - Technology \& Engineering.

[25] Wise, W.R., Proctor, J.F., 1965. Explosion Containment Laws for Nuclear Reactor Vessels. U.S. Naval Ordnance Laboratory Report NOLTR-63-140. 